\title{
Hydrogen-Natural Gas Mixture Leak Detection Using Reduced Order Modelling
}

\author{
Norazlina Subani, Norsarahaida Amin*, Baba Galadima Agaie \\ Department of Mathematical Sciences, Faculty of Science, UniversitiTeknologi Malaysia, Skudai, Johor, Malaysia \\ Email address: \\ norazlina.subani@yahoo.com (N. Subani),norsarahaida@utm.my (N. Amin), babaagaie@yahoo.com (B. G. Agaie)
}

To cite this article:

Norazlina Subani, Norsarahaida Amin, Baba Galadima Agaie. Hydrogen-Natural Gas Mixture Leak Detection Using Reduced Order Modelling. Applied and Computational Mathematics. Vol. 4, No. 3, 2015, pp. 135-144. doi: 10.11648/j.acm.20150403.16

\begin{abstract}
Transient pressure wave detection analysis to detect the location of leakage on a pipeline containinghydrogen-natural gas mixture is presented. The transient pressure wave is generated either by rapid or sudden closure of the downstream shut-off valve. The governing equations of unsteady, compressible and isothermal one-dimensional flow are solved using the reduced order modelling technique. The solutions obtained when the transient condition is generated using the rapid closure valve show good agreement with published results. When the sudden closure valve is considered, the transient pressure, celerity wave, mass flux and the amount of leak discharge are shown to increase when the hydrogen mass ratio is increased. The amount of leak discharge which is calculated based on the computed celerity and pressure waves is found to be dependent on the leak positions.
\end{abstract}

Keywords: Leak Detection, Pressure-Wave Analysis, Transient Flow, Hydrogen, Natural Gas, Gas Mixture, Reduced-Order Modelling, Compressible Flow

\section{Introduction}

Hydrogen could play an important role as a sustainable energy supply, since the utilization of hydrogen yields no carbon dioxide (CO2). Hydrogen is an attractive, clean flammable gas and considered as a future energy source[10]. If hydrogen is made from renewable energy sources without yielding a lot of $\mathrm{CO} 2$, it would be possible to produce and use energy with near zero emissions of greenhouse gases or air pollutants. However, hydrogen has a storage problem and it does not exist on its own. It occurs chiefly in combination with other gases such as natural gas. The transportation of natural gas and hydrogen is feasible as long as the mass ratio of hydrogen remains sufficiently low, which is in the range of $10 \%$ to $20 \%$ hydrogen by volume mixed with natural gas[21].

Mixing higher percentages of hydrogen requires special attention regarding the durability and integrity of the pipeline and functioning of end-user appliances with respect to flame stability and increased nitrogen oxide (NOx) emissions [20]. In this case, leakage detection is very important to consider because pipelines contain hazardous and flammable gas. Leakage in pipelines, can cause serious problems related not only to the environment or safety, but also to the economy [12]. Leaks also waste natural resources and create public health risk. This is because every leak is a potential entry point for toxins in the pipeline system.

Risk of leakage through pipelines is well studied for natural gas $[19,22]$, but not for hydrogen or hydrogen-natural gas mixtures. In the gas pipeline transportation system, the existing pipe is designed and constructed specifically for natural gas only. The chemical and physical properties of hydrogen differ significantly from those of natural gas. Addition of even a small quantity of hydrogen to natural gas may have an impact on the safety related to the delivery of gas and the integrity of the network. The problem of hydrogen or hydrogen-natural gas mixture release appears to be a major potential risk that should be predicted [11].

It is difficult to detect leakage in pipelines using detection sensors, especially for underground pipelines. Generally, techniques of leakage detection in pipelines are divided into external methods (hardware based methods) and internal methods (software based methods). Hardware-based methods rely mainly on the use of special sensing devices to detect fluid leakage. It depends on the type of sensors and equipment used for detection. Hardware-based methods can be further classified into acoustic monitoring $[8,15,16,17]$, optical fiber, 
cable sensor, soil monitoring, ultrasonic flow meter, and vapor sampling methods. These methods are able to detect very small leaks and the leak location, but the time taken to detectleakages is not short and the installation costs of the sensors are expensive.

Software-based methods have software programs based on mathematical modelling. The algorithms continuously monitor the state of pressure, temperature, flow rate and other pipeline parameters [18]. Leaks can be detected based on the evolution of these parameters. Different software-based approaches to detect leaks are the volume or mass balance methods, transient pressure wave detection analysis[9,11, 12], and real time model ling methods $[1,7,13]$.

Aamo et al. [1] and Billmann and Isermann [7] determined leakage in pipelines by using real time modelling. They designed an observer with friction adaptation to detect, quantify and locate the leak. Later, Hauge et al. [13] improved the development of leak detection by varying time boundary conditions. They simulated leak detection for both liquid and gas flow. However, this method is very expensive because it requires extensive instrumentation for collecting data in real time. The models employed are complex and require a trained user.

Elaoud and Hadj-Taïeb[11] and Elaoud et al. [12] determined the leakage of hydrogen-natural gas mixture in pipelines. The transient pressure wave technique is used to locate the leak position started by the rapid closure of a downstream shut-off valve. Brunone et al. [9] also used this technique to determine the leakage in awater pipeline system. The transient pressure wave analysis is available for all transient flows. The advantage of this technique is that a small leak can be detected at a very low cost.

The purpose of this paper is to determine and locate leakage in the piping system of hydrogen-natural gas mixture based on transient pressure wave analysis. The effect of hydrogen mass ratio on the transient flow of hydrogen-natural gas mixture and leak discharge is analyzed. The transient flow is created by the sudden closure of a downstream shut-off valve. The governing equations are solved by the reduced order modelling (ROM) technique $[2,3,4]$. This numerical method is often used as a computational technique to solve transient flow problem because of less computational time and cost [5, $6]$.

\section{Mathematical Formulation}

The governing equations consist of two coupled non-linear hyperbolic partial differential equations. The flow is assumed to be one dimensional, isothermal, compressible and includes transient condition. The fluid is assumed to be a homogeneous mixture of hydrogen and natural gas.

\subsection{Governing Equation}

From the principle of conservation of mass and momentum laws, the governing equations for transportation of hydrogen-natural gas mixture in a horizontal pipeline are given by:

$$
\begin{gathered}
\frac{\partial \rho}{\partial t}+\frac{\partial(\rho u)}{\partial x}=0 \\
\frac{\partial(\rho u)}{\partial t}+\frac{\partial\left(\rho u^{2}+P\right)}{\partial x}+\frac{f \rho u|u|}{2 D}=0
\end{gathered}
$$

where $\rho$ is defined as density, $u$ is the gas velocity, $P$ is the pressure, $f$ is the coefficient of friction, and $D$ is the diameter of the pipeline where the modulus sign is to ensure that the frictional force shall always act opposite to the direction of motion.

Equations (1) and (2) form a system of two non-linear partial differential equations of hyperbolic type. Pressure $P$ and velocity $u$ are considered as the main variables of the flow.

\subsection{Hydrogen-Natural Gas Mixture Equation}

For hydrogen-natural gas mixture, the hydrogen mass ratio will be used in determining the mixture density, where the mass ratio of the mixture is given as:

$$
\varphi=\frac{m_{h}}{m_{h}+m_{g}}
$$

where $m_{g}$ and $m_{h}$ are defined as the mass of natural gas and hydrogen, respectively.

The density of hydrogen and natural gas according to isentropic laws is written as:

$$
\begin{aligned}
& \rho_{h}=\rho_{h_{0}}\left(\frac{P}{P_{0}}\right)^{\frac{1}{n}} \\
& \rho_{g}=\rho_{g_{0}}\left(\frac{P}{P_{0}}\right)^{\frac{1}{n^{\prime}}}
\end{aligned}
$$

where each $\rho_{h_{0}}$ represents the initial density of hydrogen and $\rho_{g_{0}}$ represents natural gas. $P$ is the transient pressure and $P_{0}$ is permanent pressure.

To solve the governing equations (1) and (2) numerically, the density of the hydrogen-natural gas mixture $\rho$ must be expressed according to the gaspres sure $P$. The expression of the average density of the mixture is defined according to the mass ratio $\varphi$, given by:

$$
\begin{aligned}
\rho & =\left[\frac{\varphi}{\rho_{h}}+\frac{(1-\varphi)}{\rho_{g}}\right]^{-1} \\
& =\left[\frac{\varphi}{\rho_{h_{0}}}\left(\frac{P}{P_{0}}\right)^{\frac{1}{n}}+\frac{(1-\varphi)}{\rho_{g_{0}}}\left(\frac{P}{P_{0}}\right)^{\frac{1}{n^{\prime}}}\right]^{-1}
\end{aligned}
$$

For compressible flow, the celerity of pressure wave is defined as: 


$$
c^{2}=\left(\frac{\partial P}{\partial \rho}\right)_{s}, \text { thus } c=\left(\frac{\partial \rho}{\partial P}\right)_{s}^{-\frac{1}{2}}
$$

where the subscript $s$ denotes the condition of constant entropy.

By taking the derivative of (5) with respect to $P$, the celerity pressure wave (6) yields:

$$
\begin{aligned}
c= & {\left[\frac{\varphi}{\rho_{h_{0}}}\left(\frac{P}{P_{0}}\right)^{\frac{1}{n}}+\frac{(1-\varphi)}{\rho_{g_{0}}}\left(\frac{P}{P_{0}}\right)^{\frac{1}{n^{\prime}}}\right] \times } \\
& {\left[\frac{1}{P}\left\{\frac{\varphi}{n \rho_{h_{0}}}\left(\frac{P}{P_{0}}\right)^{\frac{1}{n}}+\frac{(1-\varphi)}{n^{\prime} \rho_{g_{0}}}\left(\frac{P}{P_{0}}\right)^{\frac{1}{n^{\prime}}}\right\}\right]^{-\frac{1}{2}} }
\end{aligned}
$$

\section{Numerical Solution}

To solve the governing equations (1) and (2) numerically, ROM is employed $[2,3,4,5,6]$.The governing equations (1) and (2) aretransformed in the flux vector form:

$$
\frac{\partial Q}{\partial t}+\frac{\partial E(Q)}{\partial x}-H(Q)=0
$$

where

$$
Q=\left[\begin{array}{c}
\rho \\
\rho u
\end{array}\right], E(Q)=\left[\begin{array}{c}
\rho u \\
\rho u^{2}+c^{2} \rho
\end{array}\right] \text { and } H(Q)=\left[\begin{array}{c}
0 \\
-\frac{f \rho u|u|}{2 D}
\end{array}\right] .
$$

\subsection{Implicit Steger-Warming Flux Vector Splitting Scheme}

The implicit Steger-Warming flux vector splitting method (FSM) is used as the numerical scheme. Finite difference method (FDM) is used to discretize equation (8) and to obtain FSM scheme [14]. The resulting FSM scheme in delta notation is:

$$
\begin{gathered}
-\left[\frac{\Delta t}{\Delta x} A_{i-1}^{n(+)}\right] \Delta Q_{i-1}+\left[\frac{\Delta t}{\Delta x} A_{i+1}^{n(-)}\right] \Delta Q_{i+1}+ \\
{\left[I+\frac{\Delta t}{\Delta x}\left(A_{i}^{n(+)}-A_{i}^{n(-)}\right)-\Delta t B_{i}^{n}\right] \Delta Q_{i}=} \\
-\frac{\Delta t}{\Delta x}\left[E_{i}^{n(+)}-E_{i-1}^{n(+)}+E_{i+1}^{n(-)}-E_{i}^{n(-)}\right]+\Delta t H_{i}^{n}
\end{gathered}
$$

where

$$
\Delta Q=Q^{n+1}-Q^{n}
$$

and $I$ is the identity matrix, $A$ and $B$ are the Jacobian matrices defined as:

$$
A=\frac{\partial E(Q)}{\partial Q}, B=\frac{\partial H(Q)}{\partial Q}
$$

From (9), the Jacobian matrix $A$ is split into two parts (positive and negative), denoted by $A^{+}$and $A^{-}$:

$$
\begin{gathered}
A^{+}=\left[\begin{array}{cc}
\frac{c^{2}-u^{2}}{2 c} & \frac{u+c}{2 c} \\
\frac{(u+c)^{2}(c-u)}{2 c} & \frac{(u+c)^{2}}{2 c}
\end{array}\right] \\
A^{-}=\left[\begin{array}{cc}
\frac{u^{2}-c^{2}}{2 c} & \frac{c-u}{2 c} \\
\frac{(u+c)(c-u)^{2}}{2 c} & -\frac{(c-u)^{2}}{2 c}
\end{array}\right]
\end{gathered}
$$

while $E^{+}$and $E^{-}$are the positive and negative parts of the flux vector $E$ given by:

$$
E^{+}=\left[\begin{array}{c}
\frac{\rho(u+c)}{2} \\
\frac{\rho(u+c)^{2}}{2}
\end{array}\right], E^{-}=\left[\begin{array}{c}
\frac{\rho(u-c)}{2} \\
\frac{\rho(u-c)^{2}}{2}
\end{array}\right]
$$

When (9) is applied to each grid point, a block tridiagonal of systems of algebraic equations is obtained. The system equationis solved at each time step, and results in $\Delta Q$. The values of $Q$ at the next time step can be calculated using (10).

To perform the eigenanalysis and to construct ROM based on the flow eigenmodes, it is necessary to linearize the finite difference equation in (9). The linearization can be achieved by assuming steady state results in which stability perturbation is used to obtain the transient solution at each nodal point. For linearization, the flow field variables at each time step are considered as:

$$
Q^{n+1}=Q^{0}+\hat{Q}^{n+1}
$$

where $Q^{0}$ represents the corresponding steady state values and $\hat{Q}$ represents the perturbation values.

Substituting (14) into (9) yields:

$$
\begin{aligned}
& -\left[\frac{\Delta t}{\Delta x} A_{i-1}^{0(+)}\right] \hat{Q}_{i-1}^{n+1}+\left[\frac{\Delta t}{\Delta x} A_{i+1}^{0(-)}\right] \hat{Q}_{i+1}^{n+1} \\
& +\left[I+\frac{\Delta t}{\Delta x}\left(A_{i}^{0(+)}-A_{i}^{0(-)}\right)\right] \hat{Q}_{i}^{n+1}-\Delta t B_{i}^{0} \hat{Q}_{i}^{n+1} \\
& =\hat{Q}_{i}^{n}
\end{aligned}
$$

The above linearization equation (15)is represented in the form of eigenvalue problem:

$$
W^{0} \hat{Q}^{n+1}=I \hat{Q}^{n}+V^{n+1}
$$

where

$$
V^{n+1}=-\left[\frac{\Delta t}{\Delta x} A_{i-1}^{0(+)}\right] \hat{Q}_{i-1}^{n+1}+\left[\frac{\Delta t}{\Delta x} A_{i+1}^{0(-)}\right] \hat{Q}_{i+1}^{n+1}
$$


and

$$
W^{0}=\left[I+\frac{\Delta t}{\Delta x}\left(A_{i}^{0(+)}-A_{i}^{0(-)}\right)\right]-\Delta t B_{i}^{0}
$$

and $V$ is defined as a vector consisting of the imposed values of the boundary conditions and $W^{0}$ is the coefficient of the matrixrepresented by the left hand side terms of (16). The finite difference equation (15) is the linearized form of FSM.

\subsection{Reduced Order Modelling Construction}

To construct the ROM technique, the zero eigenvalue in the eigen system of matrix are required. For zero forcing function $V$, we need to consider homogeneous part of (16) by setting:

$$
\hat{Q}_{i}=x_{i} \exp \left(\lambda_{i} t\right) \alpha_{i} \exp \left(i z_{i} x\right)
$$

where $\lambda_{i}$ represents eigenvalues, $x_{i}$ is eigenvector and $z_{i}=\exp \left(\lambda_{i} \Delta t\right)$.

Equation (17)is used to obtain the following generalized eigenvalue problem. Then, the diagonal matrix which contains the eigenvalues and eigenvectors can be written as:

$$
z_{i} W^{0} x_{i}=I x_{i}
$$

The general form of equation (18) can be written as:

$$
Z W^{n} X=I X
$$

where $Z$ represents the diagonal matrix of eigenvalues at each time step and $X$ is the matrix with column that are the right eigenvector.

On the other hand, the left eigenvectors satisfy the following relation:

$$
\left(W^{0}\right)^{T} Y Z=I Y
$$

where $Y$ is the matrix with rows that are the left eigenvectors.

If the eigenvectors are suitably normalized, they satisfy the following orthogonallity conditions:

$$
Y^{T} W^{0} X=I \text { and } Y^{T} I X=Z
$$

For analysis of eigen mode based on the time basis, (17) reduces the gas flow behavior to the sum of individual nodes:

$$
\hat{Q}_{i}=x_{i} \exp \left(\lambda_{i} t\right)
$$

Equation (22) can be written in general form as:

$$
\hat{Q}=X \hat{c}
$$

where $\hat{c}$ is the vector of normal node coordinate.

From (23), $\hat{Q}$ and $\hat{c}$ denoted by:

$$
\hat{Q}=\left[\begin{array}{c}
Q_{1} \\
Q_{2} \\
\vdots \\
Q_{n}
\end{array}\right] \text { and } \hat{c}=\left[\begin{array}{c}
\hat{c}_{1} \\
\hat{c}_{2} \\
\vdots \\
\hat{c}_{n}
\end{array}\right]
$$

Multiplying(23) by $Y^{T} W^{0}$ we have:

$$
\hat{c}=Y^{T} W^{0} Q
$$

Substituting (21) and (16), then multiplying by $Y^{T}$ gives a set of $N$ uncoupled equations for the nodal coordinates $\hat{c}$ :

$$
\hat{c}^{n+1}=Z \hat{c}^{n}+Y^{T} V^{n+1}
$$

Since the orthogonallity conditions are satisfied, the eigen mode can be retained to construct ROM by using (26). Using (23) and (26), the space time iteration of unknown matrix is reduced to order one. The results are reliable and steady if the zero and negative eigenvalues are removed. Then, the transient flow of hydrogen-natural gas mixture now is analyzed using (23) and (26) which contains the results of nonzero and positive eigenvalues.

\section{Leakage Detection}

The problem is focused on transients occurring in a single pipeline with a constant pressure reservoir at the upstream end and a sudden closure valve at the downstream end as illustrated in Fig. 1 [12].

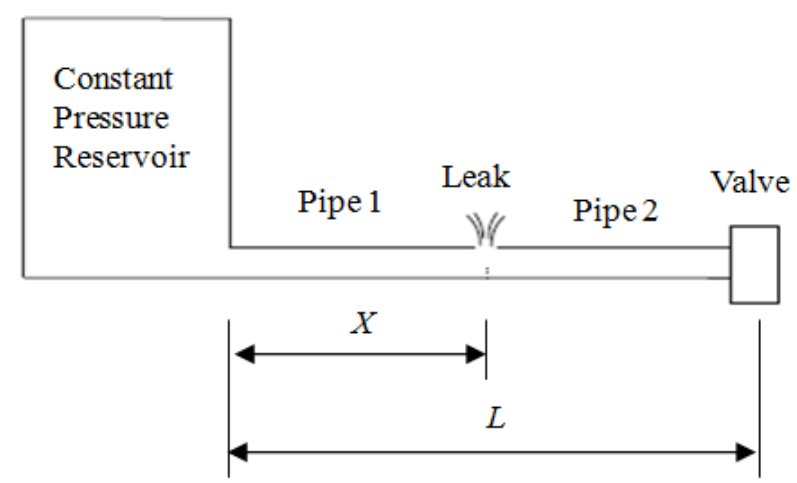

Figure 1. Hydrogen-natural gas mixture installation with leakage [12].

A single leak is supposed to exist at an intermediate section of the pipeline and located at a distance $X$ from the reservoir. The pipeline with a length $L$ is divided into two segments, which is Pipe 1, from the reservoir to the leak, and Pipe 2 from the leak to the valve.

\subsection{Leak Modelling}

The leak orifice can be simulated based on the flow rate through a small orifice between the high-pressure pipeline and the environment. According to Elaoud et al. [12], the discharged flow $Q_{l}$ from the orificecan be calculated by the given equation: 


$$
Q_{l}=\rho_{\left(P_{l}\right)} C_{d} A_{l} \sqrt{2 P_{l} / \rho_{\left(P_{l}\right)}}
$$

where $C_{d}$ is a discharge coefficient, $A_{l}$ is the orifice area of the leak, $P_{l}$ and $\rho_{\left(P_{l}\right)}$ are the pressure and the density at the leak position.

The analysis of transient pressure wave of hydrogen-natural gas mixture is based on the properties of transmission and reflection of pressure wave caused by sudden closure at downstream valve. When the initial pressure wave reaches the leak, it will produce a reflection when it arrives back at the downstream end section. The time difference between the initial transient wave and the reflected wave can be then measured and position of leakage $X$ in the pipeline can be calculated by:

$$
X=L-\frac{\Delta t_{l} c}{2}
$$

where $X$ is the distance to the leak from the upstream end section, $\Delta t_{l}$ is the time difference between the initial transient wave and the reflected wave and $c$ is transient celerity wave.

By considering partial reflection of pressure waves, which take place at the leak, the discharged behavior of the leak may be obtained by:

$$
Q_{l_{0}}=\frac{A}{c}\left(\Delta P_{0}-\Delta P_{l}\right)
$$

where $Q_{l_{0}}$ is the leak discharge for transient flow, $\Delta P_{0}$ and $\Delta P_{l}$ are pressure rises produced by the sudden closure of the downstream end valve assuming the pipe to be intact or containing a leak, respectively.

\subsection{Initial and Boundary Conditions}

The initial conditions at time $t=0$ can be determined by computing the solution of the following system of ordinary differential equations deduced from (1) and (2). The initial conditions at $t=0$ are described as:

$$
\begin{gathered}
\frac{\partial \rho u}{\partial x}(x, 0)=0 \\
\frac{\partial\left(\rho u^{2}+P\right)}{\partial x}(x, 0)+\frac{f \rho u|u|}{2 D}=0
\end{gathered}
$$

For boundary conditions, the condition is given by the reservoir constant pressure at the upstream end $(x=0)$,

$$
P_{1}(t, 0)=P_{0}
$$

The transient flow is created by the fast closure of the valve at the downstream end $(x=L)$. At this point, the discharge flow of leakage is given by:

$$
Q_{2}(t, L)=0
$$

At the leak point, (27) is implemented as the internal boundary condition. The two relationships that related the upstream pressure and discharge flow to the downstream pressure and discharge flow are:

$$
\begin{gathered}
P_{1}=P_{2}=P_{p} \\
Q_{1}=Q_{2}+\rho_{\left(P_{p}\right)} C_{d} A_{l} \sqrt{2 P_{p} /} \rho_{\left(P_{p}\right)}
\end{gathered}
$$

where $Q_{1}$ and $Q_{2}$ are the discharge at upstream and downstream of the leak, respectively and $\rho_{\left(P_{l}\right)} C_{d} A_{l} \sqrt{2 P_{l} /} \rho_{\left(P_{l}\right)}$ is the leak flow rate.

\section{Results and Discussion}

To locate the leak by the transient flow analysis of hydrogen-natural gas mixture, an iron pipe $0.4 m$ in diameter and $600 \mathrm{~m}$ long is considered. This pipe is connected to a constant pressure reservoir at the upstream end, while the sudden closure valve is at the downstream end. A mass flow $Q_{0}=55 \mathrm{Kg} / \mathrm{s}$ at a static temperature $T=15^{\circ} \mathrm{C}$ and an absolute pressure $P=35 \mathrm{bar}$ are assumed. The properties of hydrogen and natural gas used in the calculations are those of Elaoud et al. [12]. The steady state solutions are presented in Fig 2. These results are taken as the initial condition to analyze the transient flow behavior of hydrogen-natural gas mixture on leakage detection.

Fig. 2 illustrates the pressure distribution along the pipe for different values of mass ratio $(\varphi=0, \varphi=0.5$ and $\varphi=1)$. The above method of ROM is validated by comparison with the characteristics method in the case of pipes without leak. It is observed that the results obtained using ROM are in good agreement with those of the characteristics method. The numerical simulation is done for a leak located at various positions $(X=L / 3, X=L / 2$ or $X=2 L / 3)$. These graphs are plotted by assuming the pipe to be intact or with the presence of a leak at the $X$ distance from the reservoir. From Fig. 2, the results clearly show that the downstream end pressure is more important when the leak is closer to the reservoir.

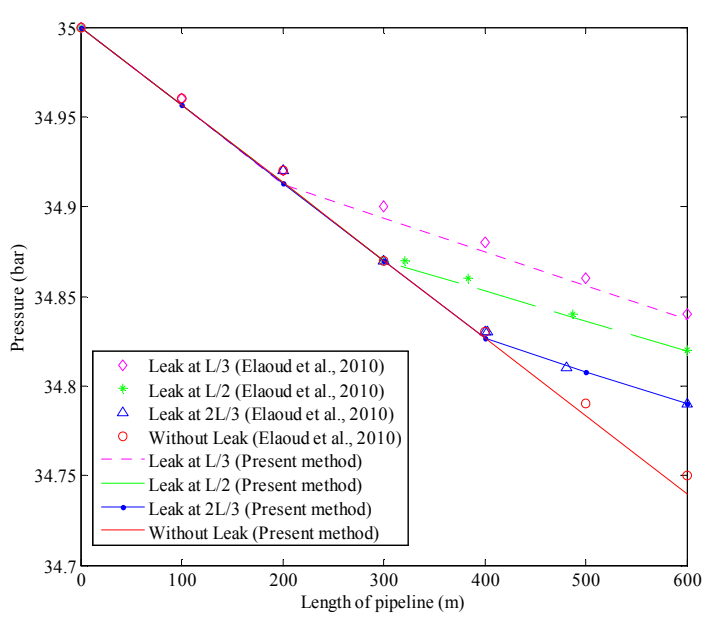

a) Natural gas $(\varphi=0)$ 


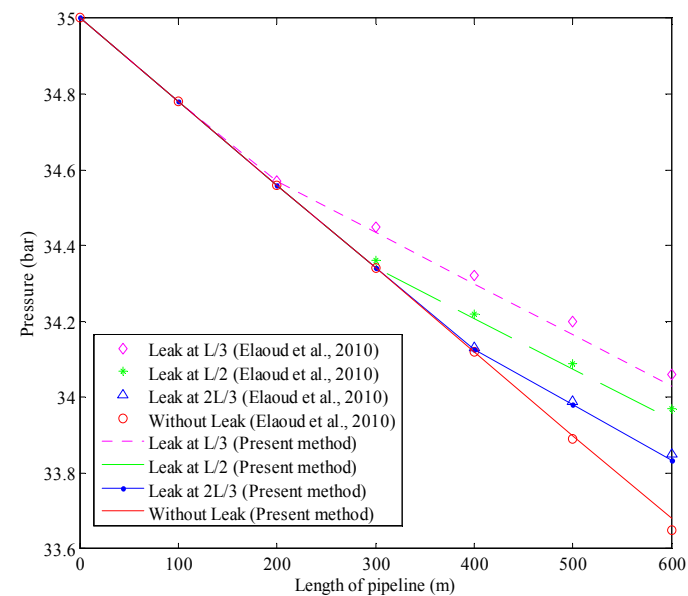

b) Hydrogen-natural gas mixture ( $\varphi=0.5$ )

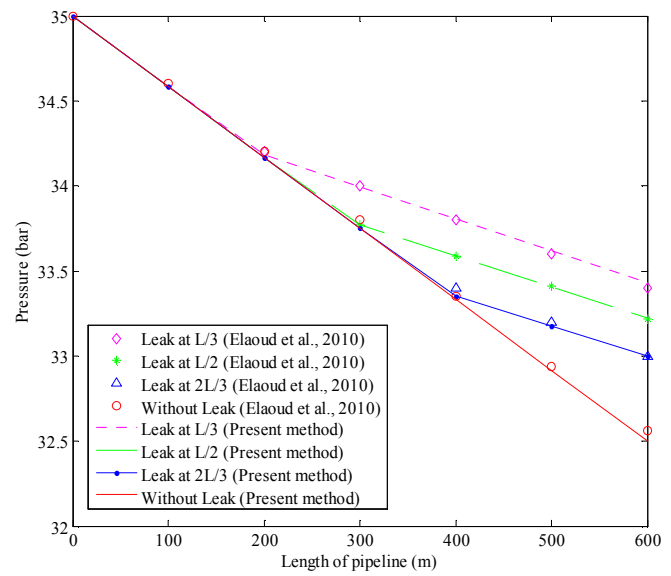

c) Hydrogen $(\varphi=1)$

Figure 2. Pressure distribution along the pipeline for different values of mass ratio $\varphi[12]$.

As indicated in Fig. 2, for an intact pipeline, the pressure is lower compared to the pipeline with the presence of leak. The pressure for hydrogen is quickly reduced compared to the natural gas and hydrogen-natural gas mixture.

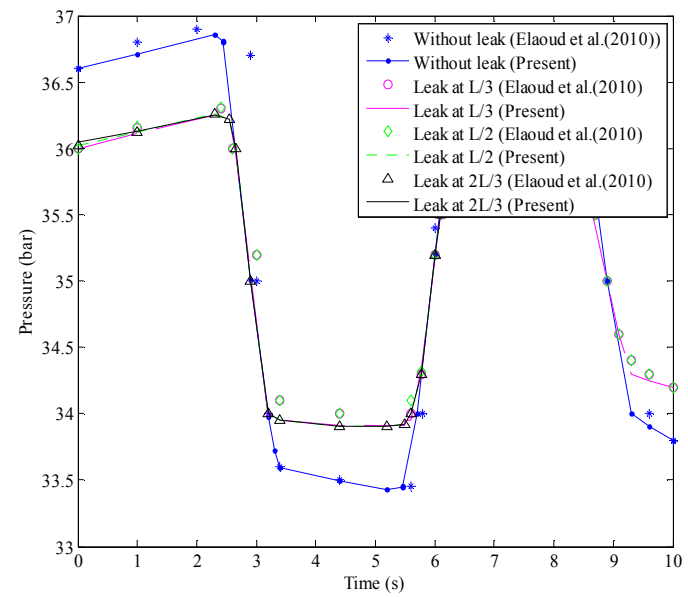

a) Natural gas $(\varphi=0)$

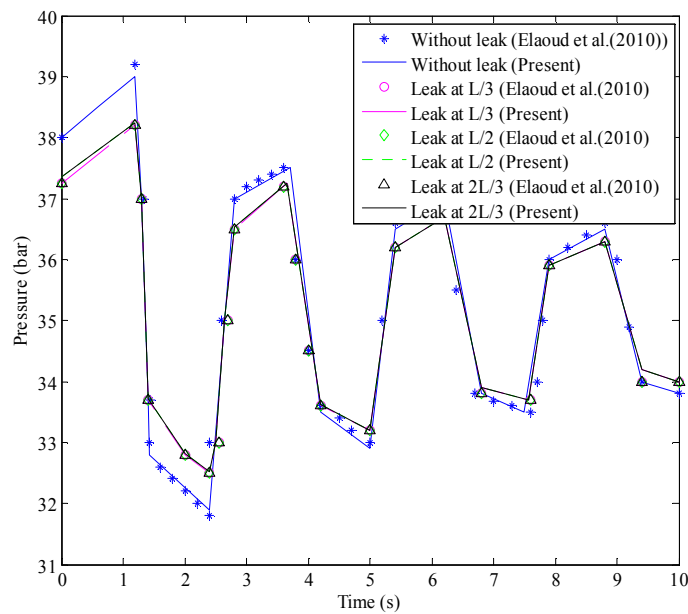

b) Hydrogen-natural gas mixture ( $\varphi=0.5$ )

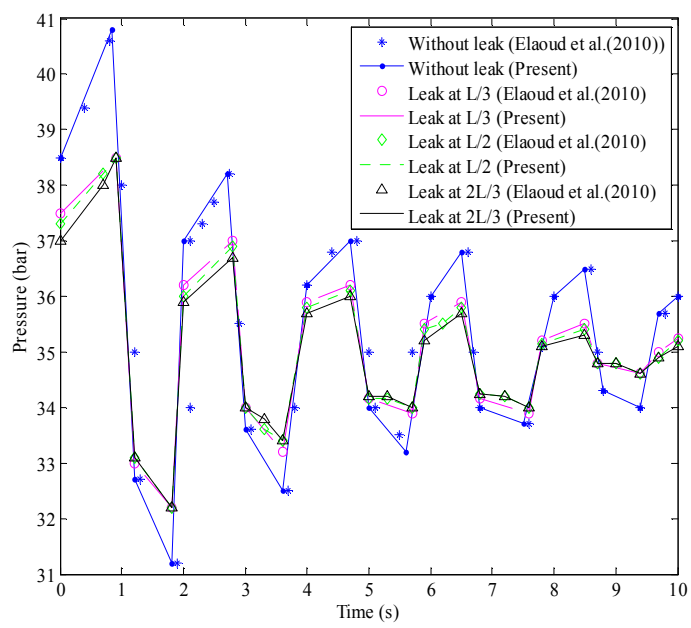

c) Hydrogen $(\varphi=1)$

Figure 3. Transient pressure distribution for the rapid closure valve at the downstream end of the pipe for different values of mass ratio $\varphi$ [12]

Fig. 3 shows the numerically obtained results for the transient pressure distribution after rapid closure of the downstream valve as a function of time and for different values of the hydrogen mass ratio $\varphi$. From Fig. 3, the results obtained are based on the properties of transmission and reflection of pressure waves provoked by the rapid closure of the downstream valve [12]. It is observed that the results obtained using ROM are in good agreement with those of the characteristics method.

In the presence of leak, the results obtained show that the increase of pressure is better in the case of hydrogen than that of natural gas. This increases the performance of energy when the gas flows in pipelines. Pressure distribution is an important parameter to calculate the discharged amount of gas released due to pipeline leakage in (27). This parameter is also needed in the detection and locates the position of leaks in the pipeline. This leak detection is based on the properties of transmission and reflection of pressure waves.

Fig. 4 shows the numerical results of the transient pressure wave distribution after the sudden closure of the downstream valve as a function of time and for different values of the mass 
ratio $\varphi$. These plots are at the downstream end section of the pipe calculated from (29). The pipe is supposed to be intact or with a leak at different positions $L$.

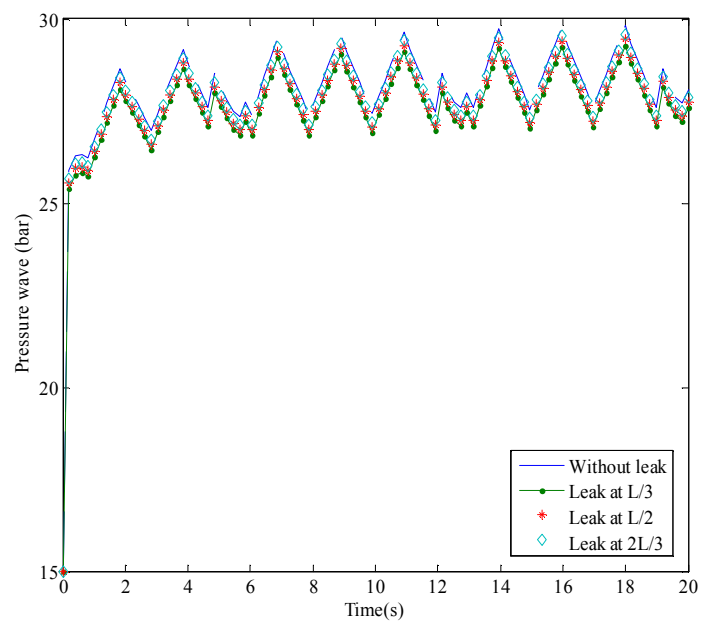

a) Natural gas $(\varphi=0)$

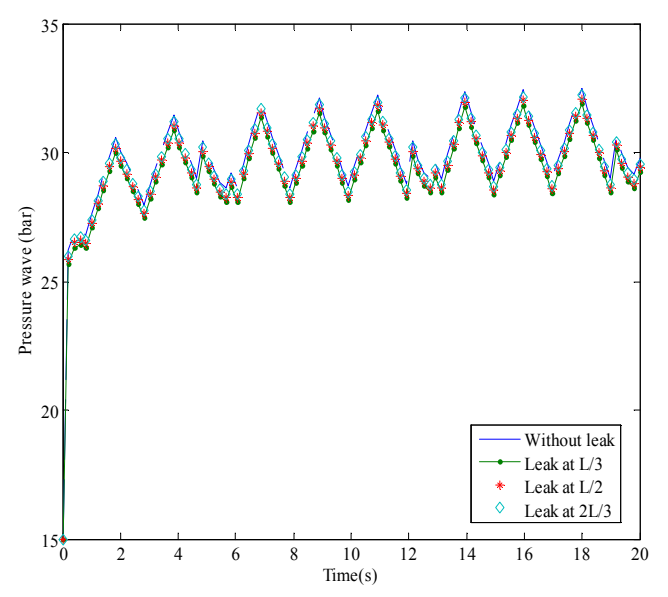

b) Hydrogen-natural gas mixture $(\varphi=0.5)$

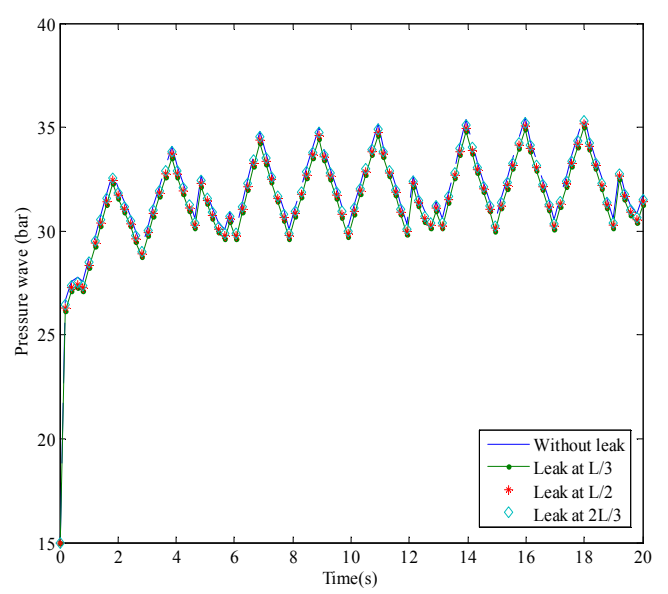

c) Hydrogen $(\varphi=1)$

Figure 4. Transient pressure wave distribution for the sudden closure valve at the downstream of the pipeline for different leak sections.

The technique proposed here for leak detection is based on the properties of transmission and reflection of pressure waves produced by the sudden closure of the downstream valve [9]. Closing the valve suddenly takes less time to reduce the pressure wave compared to rapid closure valves. Sudden closure valves should be considered to ensure the attainment of minimum pressure within a short time.

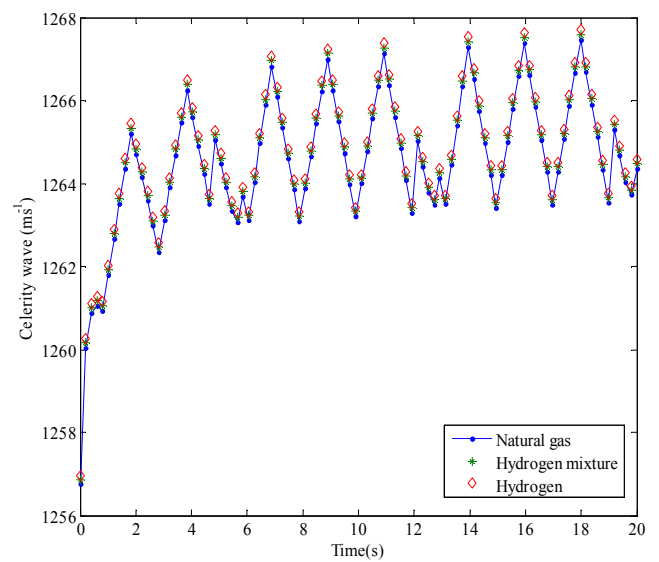

a) Located at $L / 3$

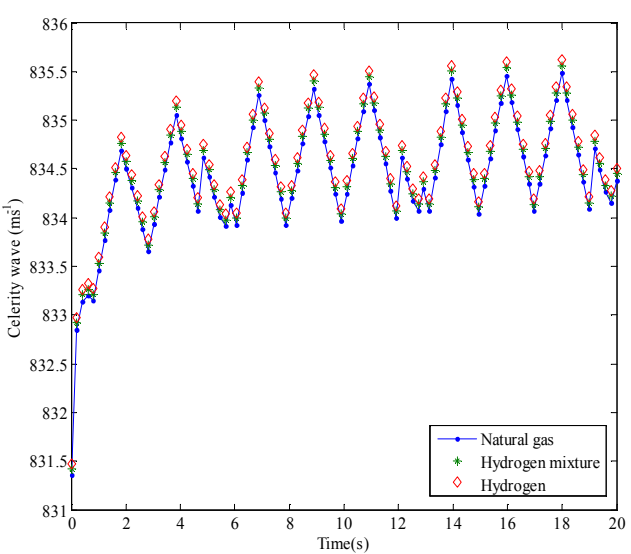

b) Located at $L / 2$

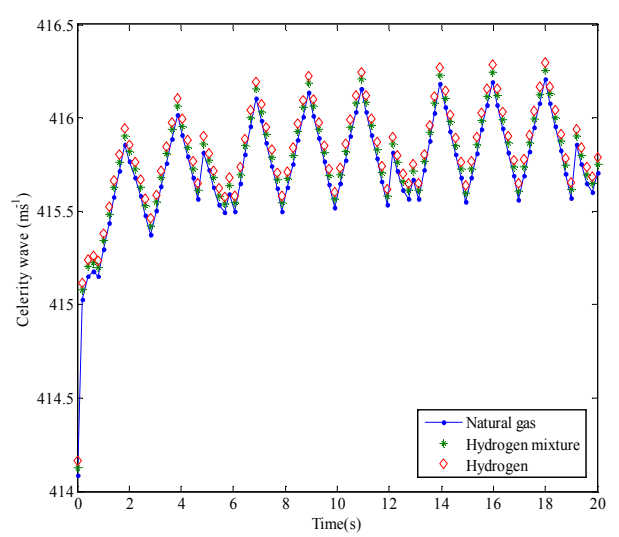

c) Located at $2 L / 3$

Figure 5. Celerity wave distributionof the pipeline at different leak sections.

Fig. 5 shows the plots of the celerity wave distribution as a function of time at different leak sections of the pipe and for different values of mass ratio $\varphi$. Ths celerity wave in (28) 
and (29) is an important parameter to calculate the location and the discharge amount of hydrogen-natural gas mixture due to leak in a pipe.

From Fig. 5, the celerity wave increases with time along the pipeline. It can be noted that the celerity wave evolution follows the same oscillation behavior after $5 s$ for all gases (natural gas, hydrogen-natural gas mixture and hydrogen). As illustrated in Fig. 5, the increase in celerity wave of hydrogen is much higher than that of natural gas. This shows that the celerity wave is more important when determining and locating leaks in the case of hydrogen $(\varphi=1)$ than that of natural gas $(\varphi=0)$. An increase in the mass ratio $\varphi$ makes it easy to locate the leakage point in the pipeline.

Fig. 6 presents the mass flux distribution as a function of time at different leak sections of the pipe and for different values of mass ratio $\varphi$. We note that the mass flux follows the same pattern for different values of $\varphi$.

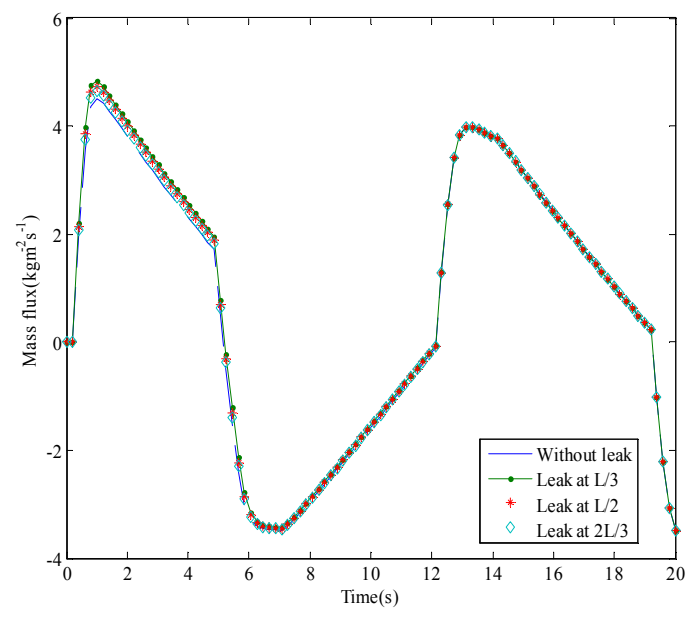

a) Natural gas $(\varphi=0)$

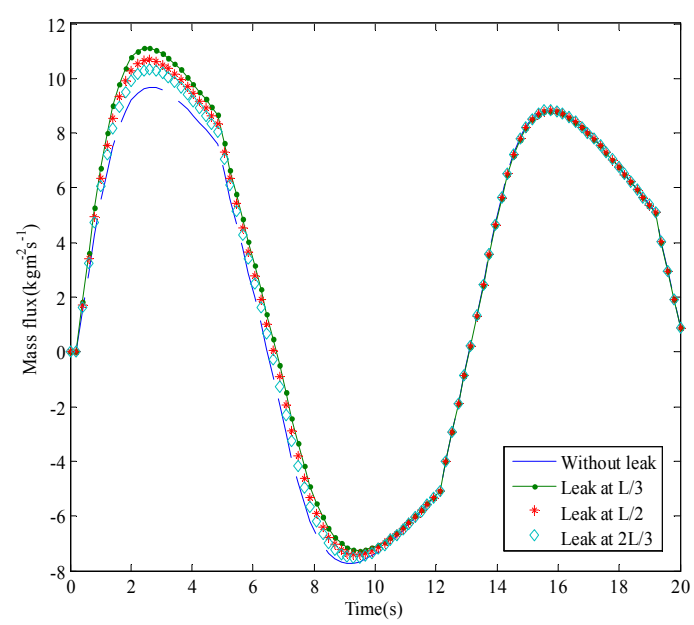

b) Hydrogen-natural gas mixture $(\varphi=0.5)$

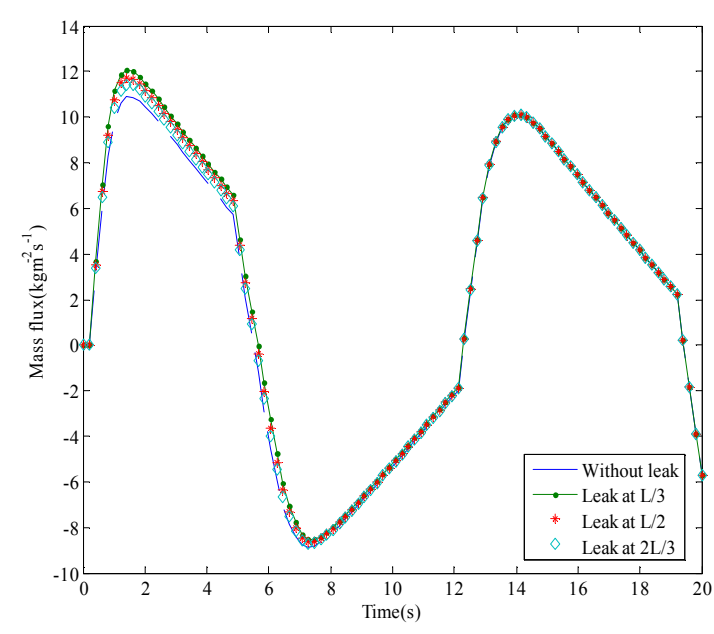

c) Hydrogen $(\varphi=1)$

Figure 6. Mass flux distribution along the pipeline at different leak sections.

As illustrated in Fig. 6, the amplitude of the mass flux oscillation decreases. In this figure, it can be shown that for the same interval, the oscillation frequency of mass flux is more important in the case of hydrogen compared to natural gas due to leak problems. Increase in the mass ratio $\varphi$ in the mass flux results in an increase in the combustion capacity. This would effect the flow transportation and the energy performance is better compared to low mass ratio $\varphi$.

From Fig. 5, assume that the lowest maximum value of celerity wave at $\Delta t_{l}$ equals: $0.62 s$, for hydrogen $(\varphi=1)$ is about $1261.8 \mathrm{~m} / \mathrm{s}$ and the leak is located at $X=L / 3$. By using (28), the leak location is predicted to be at:

$$
X=600-\frac{0.62 \times 1261.8}{2} \approx 208.84 m
$$

This result is considered acceptable since the real location of the leak is $200 \mathrm{~m}$. Table 1 presents the values of leak location for hydrogen, hydrogen-natural gas mixture and natural gas at different leakage locations.

Table 1. Computed values of leak location.

\begin{tabular}{llll}
\hline $\begin{array}{l}\text { Types of gas/ } \\
\text { Leak location }\end{array}$ & $\begin{array}{l}\text { Natural gas } \\
(\varphi=0)\end{array}$ & $\begin{array}{l}\text { Hydrogen } \\
\text { mixture } \\
(\varphi=0.5)\end{array}$ & $\begin{array}{l}\text { Hydrogen } \\
(\varphi=1)\end{array}$ \\
\hline Location at $2 L / 3(\mathrm{~m})$ & 471.29 & 471.26 & 471.23 \\
Location at $L / 2(\mathrm{~m})$ & 341.71 & 341.68 & 341.65 \\
Location at $L / 3(\mathrm{~m})$ & 209.09 & 208.97 & 208.84 \\
\hline
\end{tabular}

From Table 1, the results clearly show that the downstream end celerity wave is more important when the leak is closer to the reservoir. The results obtained show that the prediction of leak location is more precise for the leakage closer to the reservoir compared to the leakage farther from the reservoir.

Table 2 represents the amount of leak discharge at different mass ratios. The transient leak discharge amount $\left(Q_{l_{0}}\right)$ can be calculated from (29). These results were obtained by analyzing the plots of Figs. 4 and 5. The amount of leak discharge for hydrogen and the leak is located at $X=L / 3$ is predicted to 
be:

$$
Q_{l_{0}}=\frac{0.13}{1261}\left(1.20 \times 10^{6}\right)=123.71 \mathrm{~kg} / \mathrm{s}
$$

Table 2. Amount of leak discharge.

\begin{tabular}{llll}
\hline Types of gas & $\begin{array}{l}\text { Leak location } \\
(\mathbf{m})\end{array}$ & $\begin{array}{l}\Delta P_{0}-\Delta P_{l} \\
(\mathbf{N} / \mathbf{m} \mathbf{2})\end{array}$ & $\begin{array}{l}\text { Leak discharge } \\
\mathbf{( k g / s )}\end{array}$ \\
\hline Hydrogen & Leak at 2L/3 & $1.25 \times 106$ & 391.19 \\
& Leak at L/2 & $1.23 \times 106$ & 191.86 \\
& Leak at L/3 & $1.20 \times 106$ & 123.71 \\
Hydrogen- & Leak at 2L/3 & $1.23 \times 106$ & 385.02 \\
mixture & Leak at L/2 & $1.20 \times 106$ & 187.21 \\
& Leak at L/3 & $1.15 \times 106$ & 118.52 \\
Natural gas & Leak at 2L/3 & $1.15 \times 106$ & 360.07 \\
& Leak at L/2 & $1.10 \times 106$ & 171.63 \\
& Leak at L/3 & $1.03 \times 106$ & 106.19 \\
\hline
\end{tabular}

From Table 2, the results obtained clearly show that the amount of leak discharge for hydrogen is higher for all leak positions compared to hydrogen-natural gas mixture and natural gas. A lower amount of leak discharge is computed at the downstream end when the leak is closer to the reservoir ( $L / 3$ ).

\section{Conclusion}

A technique based on the analysis of transient pressure waves through hydrogen-natural gas mixture to detect and to locate leakage in a rigid pipeline has been presented. The transient pressure wave is created either by the rapid or sudden closure of a downstream shut-off valve. Sudden closure valve should be considered to ensure the attainment of minimum pressure within a short time. The boundary conditions are imposed by assuming a constant pressure reservoir upstream and a sudden closure valve downstream the pipe. The governing equations for such flows are two coupled non-linear hyperbolic partial differential equations, unsteady, compressible and isothermal one-dimensional flow which are solved using the reduced order modelling technique. It is shown that the solution obtained shows good agreement with published results when the transient condition is generated using the rapid closure valve. The results obtained when the sudden closure valve is considered show that transient pressure, celerity wave and mass flux increase when the hydrogen mass ratio $\varphi$ is increased, which shows that the presence of hydrogen enhances the mixture's burning capacity. These results are significantly influenced by the hydrogen mass ratio $\varphi$. The positions of leakage and the amount of leak discharge at the various mass ratios are also calculated. It is observed that the amount of leak discharge for hydrogen is higher for all leak positions compared to the others, while the amount of leak discharge is lower when the leak is closer to the reservoir.

\section{Acknowledgements}

Financial supports provided by Vot 00M25 and Vot 01G31, Research University Grant Scheme, Universiti Teknologi Malaysia, are gratefully acknowledged.

\section{References}

[1] Aamo, O. M., Salvesen, J., and Foss, B. A., "Observer design using boundary injections for pipeline monitoring and leak detection", International Symposium on Advanced Control of Chemical Processes, April 2-5. Gramado, Brazil: ADCHEM, pp 53-58, 2006.

[2] Agaie, B. G., "Numerical computation of transient hydrogen natural gas mixture in a pipeline using reduced order modelling", Ph. D Thesis, Department of Mathematical Sciences, Universiti Teknologi Malaysia, Skudai Johor, 2014.

[3] Agaie, B. G., and Amin, N., "The effect of water hammer on pressure oscillation of hydrogen natural gas transient flow," Applied Mechanics and Materials, 554, pp 251-255, 2014.

[4] Behbahani-Nejad, M., Haddadpour, H., and Esfahanian, V., "Reduced order modelling of unsteady flows without static correction requirement", 24th International Congress of the Aeronautical Sciences, ICAS 2004, pp 1-8, 2004.

[5] Behbahani-Nejad, M., and Shekari Y., "Reduced order modeling of natural gas transient flow in pipelines", International Journal of Engineering and Applied Sciences, 5(7), pp 148-152, 2008.

[6] Behbahani-Nejad, M., and Shekari, Y., "The accuracy and efficiency of a reduced-order model for transient flow analysis in gas pipelines", Journal of Petroleum Science and Engineering, 73, pp 13-19, 2010.

[7] Billmann, L., and Isermann, R., "Leak detection methods for pipelines", International Federation of Automatic Control, 23 (3), pp 381-385, 1987.

[8] Brunner, A. J., and Barbezat, M., "Acoustic emission monitoring of leaks in pipes for transport of liquid and gaseous media: A model experiment", Advanced Materials Research Trans. Tech. Publications, pp 13-14 and pp 351-356, 2006.

[9] Brunone, B., Ferrante, M., and Ubertini, L., "Leak analysis in pipes using transients", Second Annual Seminar on Comparative Urban Projects, June 19-23, Rome, Italy, pp 1-8, 2000 .

[10] Corbo, P., Migliardini, F., and Veneri, O., "Hydrogen fuel cells for road vehicles, green energy and technology", London: Springer-Verlag, pp 33-70, 2011.

[11] Elaoud, S., and Hadj-Taïeb, E., "Leak detection of hydrogen-natural gas mixtures in pipes using the pressure-time transient analysis", Ecologic Vehicles Renewable Energies, EVRE, Monaco, 2009.

[12] Elaoud, S., Hadj-Taïeb, L., and Hadj-Taïeb, E., "Leak detection of hydrogen natural gas mixtures in pipes using the characteristics method of specified time intervals", Journal of Loss Prevention in the Process Industries, 23, pp 637-645, 2010.

[13] Hauge, E., Aamo, O. M., and Godhavn, J. M., "Model based pipeline monitoring with leak detection", Seventh IFAC Symposium on Nonlinear Control Systems, August 22-24, Pretoria, South Africa: NOLCOS, 7 (1), pp 1-6, 2007.

[14] Hoffmann, K. A. and Chiang, S. T. "Computational Fluid Dynamics for Engineers Volume I", $4^{\text {th }}$ Edition. A Publication of Engineering Education System. Wichita, Kansas USA, 2000. 
[15] Hunaidi, O., and Chu, W. T., "Acoustical characteristics of leak signals in plastic water distribution pipes", Applied Acoustics, 58, pp 235-254, 1999.

[16] Hunaidi, O., Chu, W., Wang, A., and Guan, W., "Detecting leaks in plastic pipes", Journal AWWA, 92 (2), pp 82-94, 2000.

[17] Hunaidi, O., Wang, A., Bracken, M., Gambino, T., and Fricke, C., "Acoustic methods for locating leaks in municipal water pipe networks", International Water Demand Management Conference, May 30- June 3, Dead Sea, Jordan: NRCC, pp 1-14, 2004.

[18] Jin, H., Zhang, L., Liang, W., and Ding, Q., "Integrated leakage detection and localization model for gas pipelines based on the acoustic wave method", Journal of Loss Prevention in the Process Industries, 27, pp 74-88, 2014.
[19] Turner, W. J., and Mudford, N. R., "Leak detection, timing, location and sizing in a gas pipelines", Math Compt. Modelling, 10 (8), pp 609-627, 1988.

[20] Uilhoorn, F. E., "Dynamic behaviour of non-isothermal compressible natural gases mixed with hydrogen in pipelines", International Journal of Hydrogen Energy, 34, pp 6722-6729, 2009.

[21] Veziroglu, T. N., and Barbir, F., "Hydrogen: The wonder fuel", International Journal of Hydrogen Energy, 17(6), pp 391-404, 1992.

[22] Wilkening, H., and Baraldi, D., "CFD Modelling of accidental hydrogen release from pipelines", International Journal of Hydrogen Energy, 32, pp 2206-2215, 2007. 